\title{
CENTRALITÉ ET ÉLÉMENTS PÉRIPHÉRIQUES DE LA REPRÉSENTATION SOCIALE DES CROYANCES EN AMAZONIE
}

\author{
CENTRALIDADE E ELEMENTOS PERIFÉRICOS DA \\ REPRESENTAÇÃO SOCIAL DE CRENÇAS NA AMAZÔNIA
}

\section{Rosuel Lima-Pereira ${ }^{1}$}

Résumé: Dans les sociétés amazoniennes, les croyances populaires constituent l'un des fondements de la culture locale. Les croyances populaires ont parfois des incidences à la fois sur la vie privée et sur la vie publique. Notre brève analyse sur la représentation sociale des croyances en Amazonie s'appuie sur la théorie de la centralité attribuée au psychologue social Français, JeanClaude Abric. D'après lui, une représentation sociale s'organise autour d'un noyau central entouré d'éléments périphériques. En partant de la définition de la représentation sociale comme étant un ensemble organisé et structuré d'informations, croyances, comportements et idées, notre étude sur la représentation sociale en Amazonie prend en considération le centre sous deux aspects: l'économique et le religieux. Ainsi, dans un premier moment, il sera présenté quelques éléments de l'histoire de la région amazonienne; ensuite, la question de l'identité ou des identités présentes sur le territoire sera abordé; pour terminer, il sera relevé dans cette étude, le rôle des nouvelles églises chrétiennes dans l'espace franco-brésilien.

Mots-clés: Guyane française; nouvelles églises; Brésil; Religions; Croyances

Resumo: Nas sociedades do território amazônico, as crenças populares são um dos fundamentos da cultura local. Essas crenças populares às vezes têm implicações diretas tanto na vida privada como na vida pública dos indivíduos praticantes. Nossa breve análise sobre a representação social de crenças na Amazônia é baseada na teoria da centralidade atribuída ao psicólogo social francês Jean-Claude Abric. Segundo ele, uma representação social é organizada em torno de um núcleo central cercado por elementos periféricos. Partindo da definição de representação social como um todo organizado e estruturado com informações, crenças, comportamentos e ideias, nosso estudo sobre representação social na Amazônia leva em consideração o centro segundo dois aspectos: o econômico e o religioso. Assim, em um primeiro momento, serão apresentados neste estudo alguns elementos da história da região amazônica; em seguida, a questão da identidade ou identidades presentes no território será abordada; para finalizar será destacado o papel das novas igrejas cristãs no espaço franco-brasileiro.

Palavras-chave: Guiana francesa; novas igrejas; Brasil; Religiões; Crenças.

\section{Introduction}

La croyance religieuse avant d'être un fait, est un acte culturel qui se transmet, interagit et se déploie entre les peuples. La religion, la religiosité et la pratique religieuse sont des éléments enracinés dans l'être identitaire de l'homme et plus particulièrement chez l'homme latinoaméricain. D'ailleurs, chez lui, cette praxis s'exprime en tout lieu et très souvent, sans aucune retenue. Quant à l'homme amazonien, il rejoint la manière de croire et de pratiquer des peuples autochtones, les Amérindiens, et du syncrétisme né du croisement ethnique entre les colonisateurs, l'homme européen et les capturés, l'homme africain réduit à l'esclavage. Aujourd'hui, chez les Créoles de Guyane, comme chez les Brésiliens du Nord, la pratique de la religion catholique

\footnotetext{
${ }^{1}$ Maître de conférences à l’Université de Guyane. E-mail: rdlp23@hotmail.com
} 
est un fait de longue date. Ce catholicisme est habité par des croyances populaires qui admettent l'efficacité de la sorcellerie et des forces surnaturelles. Au cours de l'histoire coloniale du Brésil mais aussi de la Guyane française, la religiosité des Caboclos et des Créoles a puisé nombre de ses traits dans des systèmes religieux traditionnels de l'Afrique subsaharienne. Ainsi, dans les sociétés amazoniennes, les croyances populaires constituent l'un des fondements de la culture locale. Les croyances populaires ont parfois des incidences tant sur la vie privée que sur la vie publique. Des individus ou des groupes, au-delà des appartenances religieuses, affichent parfois une distance voire un rejet de ces croyances et des pratiques qu'elles peuvent impliquer.

Dans notre brève analyse sur la représentation sociale des croyances en Amazonie, nous nous appuierons sur la théorie de la centralité attribuée au psychologue social Français, JeanClaude Abric (1941-2012). Selon lui, toute représentation sociale est organisée autour d'un noyau central et des éléments périphériques. Le noyau central est consensuel, normatif, fonctionnel. C'est lui, dans son rapport à la mémoire collective qui donne stabilité à la représentation sociale mais aussi aux normes d'un groupe. Quant aux éléments périphériques, ils sont là pour assurer la fonction régulatrice protégeant ainsi le noyau central. Ces éléments périphériques intègrent les nouveaux éléments de la représentation sociale sans pour autant mettre en péril son ensemble. En d'autres termes, les éléments périphériques actualisent et contextualisent la représentation sociale (ABRIC, 1976). En partant de la théorie de la centralité de Jean-Claude Abric que définit la représentation sociale comme un ensemble organisé et structuré d'informations, croyances, comportements et idées, notre étude sur la représentation sociale en Amazonie prend en considération le centre sous deux aspects: le premier, l'économique, la Guyane comme terre d'immigration socioéconomique; le second, le religieux, le christianisme et ses nouvelles ramifications comme religion dominante. Quant aux éléments périphériques, il est possible d'établir l'immigration issue des pays avoisinants et les croyances polythéistes pratiquées en Amazonie.

Dans cette étude, dans un premier moment, nous aimerions de façon succincte présenter quelques éléments de l'histoire de la région amazonienne. Nous montrerons que le flux migratoire dans cette région existe, d'ailleurs comme c'est souvent le cas, pour des raisons socioéconomiques. Il est fort de remarquer que la pauvreté et la quête des jours meilleurs sont aussi une constante chez les gens originaires du territoire amazonien.

Ensuite, en partant de la question de l'identité ou des identités présentes en Amazonie, nous voudrions savoir de quelle catégorie sociologique fait partie la population du Plateau des Guyanes. S'agit-il d'une population intégrée, assimilée ou acculturée selon une vision du noyau central, c'est-à-dire les grandes métropoles Paris et Brasília. Dans cette perspective, quel type de rapport s'établit entre le centre et la périphérie? Des rapports de force, de complaisance ou de complémentarité?

Pour terminer, nous aborderons le rôle des nouvelles églises chrétiennes et les mouvements à caractère religieux, Adventistes, Evangéliques, Pentecôtistes, qui sont souvent dans le collimateur du gouvernement. Ces nouvelles croyances, quelles places ont-elles dans le panorama de la représentation sociale? Ont-elles droit à une visibilité et de quel type? Enfin, comme exemple, nous montrerons comment s'extériorise discrètement les croyances amazoniennes, celles de rite africain et celles de rite amérindien. Ce rite est encore très vivant et sans trop de contamination extérieure dans l'espace amazonien.

\section{Fondements historiques et socio-économiques du plateau des Guyanes}

La frontière franco-brésilienne se situe dans la région connue comme le bouclier guyanais ou plateau des Guyanes qui se trouve sur la côte nord-est de l'Amérique du Sud. Ce plateau s'étend sur cinq pays: le Venezuela, le Guyana, le Suriname, la Guyane française et le nord du Brésil délimité par le fleuve Amazonas. La Guyane a été occupée par le Portugal lors de l'empire

https://periodicos.unifap.br/index.php/letras

Macapá, v. 9, n. 2, $2^{\circ}$ sem., 2019 
napoléonien. En 1900, le Conseil fédéral suisse rend un arbitrage définitif et fixe la frontière franco-brésilienne sur l'Oyapock. En 1946, la Guyane obtient le statut de département français et cette logique cherche désormais à structurer le train de de vie des hommes du pays, en essayant ainsi de respecter leurs caractères originaux.

La Guyane est le plus vaste département français avec ses $83.534 \mathrm{~km}^{2}$; il est aussi

l'un des moins peuplés avec la densité la plus basse: 277.249 habitants et 3 habitants par $\mathrm{km}^{2}$. Une fois la capitale, Cayenne, quittée et les villes limitrophes, les communes de la Guyane sont de vastes territoires de plusieurs milliers de $\mathrm{km}^{2}$ où vivent 20.000 personnes pour les plus peuplés et 140 personnes pour les moins peuplé. La Guyane est certes partie intégrante de la France, mais sur le plan de la foi, les Guyanais rejoignent celles pratiquées par les peuples caribéens et amazoniens. Bien que fortement christianisée, la pratique de la religion en Guyane est habitée par des croyances populaires qui admettent l'efficacité de la magie. On y a recours afin de trouver une aide pour les problèmes matériels du quotidien.

En Guyane, la religion reste relativement présente dans la vie des individus car, bien souvent, les gestes magico-religieux accompagnent le quotidien des Guyanais. Mêlées souvent de superstitions et de syncrétisme, pour beaucoup, ces croyances alimentent la peur. Les modes de vie modernes ont certes contribué à l'influence de ces idées dites "superstitieuses" et des pratiques qu'elles impliquent. Ces idées constituent cependant l'un des fondements de la culture religieuse, avec parfois des incidences, tant sur la vie privée que sur la vie publique. Certains historiens et sociologues parlent de « christianisme paien » pour définir ce paysage religieux particulier. Beaucoup se disent culturellement chrétiens, mais sans pour autant avoir fait une véritable conversion aux valeurs prêchées par les évangiles. Cela étant, le contexte socio-économique en Guyane reste aussi tendu étant donné que la paix sociale et le chômage sont les défis majeurs pour l'État français.

En autre, la Guyane réunit en elle des manières de vivre qui tiennent de sa double appartenance, à la fois à la nation française et au continent sud-américain. De ce fait, elle présente un visage paradoxal, d'un côté, la face d'un pays riche avec des potentialités immenses; de l'autre, des aspects d'un pays sous-développé, en raison de ses infrastructures déficientes. Quant à sa population, la Guyane reçoit une masse de migrants qui accentue et diversifie sa démographie. Des fortes différences ou des écarts économiques et sociaux existent dans les pays du plateau des Guyanes mais il est vrai aussi que des stratégies de croissances sont possibles.

Quant à la démographie, la Guyane est caractérisée par le métissage, le multiculturalisme et le plurilinguisme. Cette démographie, sous ses aspects socio-économiques est dualiste. D’un côté, il y a une démographie légale avec des secteurs de production, de développement et de fonctionnement appuyés par l'État français. De l'autre côté, il y a une démographie tous azimuts, irrégulière, avec un secteur économique de subsistance, informel et souvent exploité. Depuis les années 2000, le gouvernement lutte contre l'immigration clandestine. En Guyane, les contrôles de police se sont accentués, les lois se sont durcies et les reconduites à la frontière ont augmenté. Des Surinamiens, des Haïtiens et en troisième lieu, des Brésiliens constituent les principales communautés visées par cette politique de contrôle migratoire.

En somme, il est possible de dire qu'en Amazonie, la nature avec ses fleuves, les hommes avec leurs comportements culturels et mentaux propres à cette vaste région, se déplacent, créent des lieux de vie qui rendent très difficile une analyse approfondie faute d'une plus grande approche sur le terrain. L'univers transfrontalier franco-brésilien est très riche puisque le fleuve Oyapock demeure encore, et avec la construction du pont, un endroit où le flux humain passe, accoste et vit sans souci juridique ou politique.

\section{Interactions et acquisitions socioreligieuses dans le contexte amazonien}

En France, la loi de 1905 dans son article 1e reconnaît la liberté religieuse: "La République

https://periodicos.unifap.br/index.php/letras

Macapá, v. 9, n. 2, $2^{\circ}$ sem., 2019 
assure la liberté de conscience. Elle garantit le libre exercice des cultes sous les seules restrictions édictées ci-après dans l'intérêt de l'ordre public". Elle prolonge ainsi l'article 10 de la Déclaration des droits de l'homme et du citoyen de 1789 qui consacre la liberté d'opinion, même religieuse. La Convention européenne des droits de l'homme prévoit également dans son article 9 que la liberté de manifester sa religion ou ses convictions ne peut faire l'objet d'autres restrictions que celles qui, prévues par la loi, constituent des mesures nécessaires, dans une société démocratique, à la sécurité publique, à la protection de l'ordre, de la santé ou de la morale publiques, ou à la protection des droits et libertés d'autrui. Sont ainsi garanties la liberté de conscience et la liberté de manifester son appartenance religieuse. La liberté religieuse suppose la liberté pour chacun d'exprimer sa religion, celle de la pratiquer et celle de l'abandonner, dans le respect de l'ordre public. Ceci implique notamment pour l'État et les services publics la neutralité face à toutes les religions et à toutes les croyances. L'État, se doit de rendre possible l'exercice et la pratique du culte. Le libre exercice du culte peut avoir des conséquences dans la vie sociale et nécessiter des adaptations du droit commun. L'État peut parfois adopter des réglementations spécifiques afin de garantir la liberté religieuse et, dans certains cas, proscrire les prescriptions religieuses. En somme, en France, la liberté religieuse et la laïcité vont de pair car la laïcité repose sur trois principes: la liberté de conscience et celle de manifester ses convictions dans les limites du respect de l'ordre public, la séparation des institutions publiques et des organisations religieuses, et l'égalité de tous devant la loi quelles que soient leurs croyances ou leurs convictions.

Si tous sont égaux devant la loi, la figure de l'immigrant dans la représentation sociale guyanaise, en dehors de la réputation d'être responsable et source de malheurs sociaux, est empreinte de la reconnaissance de son savoir-faire et de sa disponibilité pour travailler. Quant aux croyances présentes aujourd'hui en Amazonie, nous pouvons les partager, grosso modo, en deux grands axes religieux: le christianisme, avec le catholicisme et les autres églises chrétiennes apparues au long du XXe siècle; les croyances fétichistes et animistes d'origine africaine et amérindienne. En Amérique, au cours des siècles, Indiens et Africains sont obligés de s'enfuir aux fonds des forêts en raison des persécutions du colonisateur et des missionnaires. En Amazonie, tandis que les Indiens vivent sur les confins des terres pour échapper à l'emprise du clergé catholique, les créoles et les caboclos, à leur tour, dans la période qui a suivi l'abolition de l'esclavage en juin 1848, subissaient l'influence religieuse catholique (Marchand-Thébault, 1960). Les mariages et les premières communions s'accroissent de même que la scolarisation des jeunes dans des écoles chrétiennes.

Entre le Brésil et la Guyane, dans l'espace du plateau des Guyanes, les représentations sociales sont similaires, mais aussi opposées. Lorsqu'il s'agit des croyances religieuses dans l'espace frontalier, ces représentations tendent à se déployer et à s'homogénéiser. Dans cet espace se côtoient les trois ethnies, les Blancs, les Noirs et les Amérindiens. Chaque ethnie a son histoire, possède ses traditions et ses croyances respectives, c'est-à-dire, des croyances d'origine judéochrétienne, animiste et panthéiste. L'homme amazonien, en tant qu'être croyant, nous pousse à poser aujourd'hui la question suivante: comment le noyau central perçoit et accueille-t-il l'homme amazonien issu de la périphérie? Périphérie qu'on peut définir comme étant à la fois sociale, culturelle et surtout économique.

Le Plateau des Guyanes continue d'être un territoire faiblement peuplé avec des frontières très perméables ce qui le rend très attirants pour les pays avoisinants. La migration brésilienne, comme d'ailleurs celle des autres pays sud-américains, caribéens, chinois, vietnamien ou syrolibanais, était souvent au cours du XXe siècle, le résultat d'une politique de l'État français. Ce n'est qu'au cours des trente dernières années que ce flux migratoire est devenu aléatoire et spontané. Historiquement, l'immigration dans le Plateau des Guyanes, et spécialement la présence des Brésiliens sur le sol guyanais est de longue date et, sous certains aspects, elle s'avère encombrante pour ses habitants. Parfois, le malaise guyanais traduit les contradictions de ce voisinage qui s'exprime par une accusation généralisée: l'immigrant est responsable de tous les maux du pays. Il

https://periodicos.unifap.br/index.php/letras

Macapá, v. 9, n. 2, $2^{\circ}$ sem., 2019 
est à l'origine de la délinquance, de l'alcoolisme, de la prostitution, du trafic de stupéfiants, de l'insécurité dans tout le Département. Malgré le rejet dans les propos affichés, les risques de conflit ethnique, les rapports entre les communautés présentes dans l'espace amazonien s'inscrivent dans un processus d'intégration et d'assimilation. Enfin, la pression migratoire que subit spécialement la Guyane, surtout pour des raisons économiques tend à déséquilibrer le fonctionnement de ses structures, hôpital, école et habitation. En tout cas, l'immigration en générale met toujours en question une identité collective car elle touche au noyau central qui est l'idée qu'une communauté a d'elle-même. Il s'agit là, encore une fois de la représentation sociale et du rapport avec les éléments périphériques.

\section{Conditions et expressions socioreligieuses dans le contexte amazonien}

Dans l'espace limitrophe entre le Brésil et la France, la ville d'Oiapoque, côté brésilien, se trouve à $600 \mathrm{~km}$ de la capitale de l'État du Amapá, Macapá. Sa population est de 20962 habitants et la ville a été élevée au rang de commune, le 23 mai 1945. Sa principale fête religieuse catholique a lieu le 15 août, à la patronne de la ville, Notre Dame de la Grâce. Selon la coutume locale, la fête est marquée par le sacré et le profane, messe, procession et kermesse. Le mois de juin, les fêtes des saints patrons, Saint Antoine, Saint Jean et Saint Pierre, respectivement le 13, 24 et 29 Juin se déroulent dans tous les quartiers de la ville, où ont lieu des kermesses, des danses typiques, des défilés et des compétitions. Le deuxième dimanche d'octobre, la ville d'Oiapoque fête aussi Notre Dame de Nazareth, le Cirio ${ }^{2}$. Le Cirio de Belém, le plus grand au monde, sur le plan international, c'est la Guyane qui envoie le plus grand nombre de pèlerins, suivie par la France, le Portugal, les États-Unis et la Hollande.

Quant aux sectes ou certaines églises ou communautés de croyants, dont le rôle et la situation juridiques n'est pas encore bien défini par la loi républicaine, un rapport de la MIVILU$\mathrm{DES}^{3}$, Mission interministérielle de vigilance et de lutte contre les dérives sectaires ${ }^{4}$, signale que, 500000 Français seraient affectés par les dérives sectaires. Ce rapport affirme que les départements d'Outre-mer seraient les plus touchés et même certaines franges de l'Église catholique ne seraient pas épargnées. Des associations dites d'intérêt public contaminent de manière inquiétante le secteur professionnel; elles proposent des services, des aides sans qu'il y ait de la part des pouvoirs publics, un contrôle rigoureux. Enfin, la Mission remarque aussi que la proximité de la Guyane de l'Amérique du Nord et du Brésil la rend plus accessible aux sectes évangélistes et satanistes d'origine américaine. Par ailleurs, la Mission ajoute que, selon ses évaluations, le taux d'adhésion à une secte en Guyane est entre $20 \%$ et $25 \%$ de la population. Dans ces conditions, au moins un membre d'une famille se trouve engagé dans une secte et beaucoup sont insérés dans le dispositif administratif du Département ${ }^{5}$.

En France et spécialement dans les territoires outre-mer, des églises ou des mouvements sectaires se cachent derrière la façade d'une association caritative, sportive, éducative. Il est possible d'en répertorier quelques-unes qui ont une existence juridique en France. Dans le Journal officiel, dans les activités religieuses, spirituelles et philosophiques, 35 associations y sont présen-

\footnotetext{
${ }^{2}$ Le Círio, le Cierge de Notre-Dame de Nazareth, est une fête typique régionale. Chaque année, depuis 1793, année de la découverte d'une statue de Notre Dame, près de la rivière Murucutu, une gigantesque procession de plus d'un million et demi de personnes traverse les rues de Belém, capitale de l'Etat du Pará, pour honorer la Vierge de Nazareth. L'image de Notre Dame de Nazaré est conduite à Icoaraci où commence une procession fluviale sur la baie de Cuajarà.

${ }^{3}$ Rapport au Premier ministre, «MIVILUDES 2008, » la Documentation française, 19 mai 2009. http://www.mivi ludes.gouv.fr/IMG/pdf/Miviludes_Rapport_2008-2.pdf

${ }^{4}$ Selon le décret $n^{\circ}$ 2002-1392 du 28 novembre 2002, la MIVILUDES, Mission interministérielle de vigilance et de lutte contre les dérives sectaires est instituée auprès du Premier ministre afin de mener une action préventive et répressive des pouvoirs publics à l'encontre de ces groupes sectaires.

${ }_{5}^{5}$ MILS, Mission interministérielle de lutte contre les sectes, Rapport février 2000.
} 
tes. L'expansion des Églises évangéliques en Guyane est due à la circulation des individus d'un espace, le Brésil, à un autre, la Guyane, mais aussi à la circulation des croyants d'une Église à une autre. Cette fluidité dans l'espace religieux veut surtout afficher une opposition au catholicisme toujours dominant. Dans cette région, le plateau des Guyanes, le succès du message évangélique est étroitement lié à l'action des prédicateurs indépendants et itinérants qui se sentent chargés d'une mission divine. Cette notion de mission divine permet au prédicateur évangéliste soit d'être reconnu par l'Église dont il est le porteparole, soit de lui conférer une autorité pour fonder sa propre Église. Cette dynamique de cohésion ou de fragmentation n'affecte en rien l'unité de l'ensemble de ces églises évangéliques, adventistes, pentecôtistes. En Guyane, les pasteurs d'origine brésilienne improvisent eux aussi, leur mission religieuse et s'adaptent aux événements de la vie quotidienne locale dans le but de trouver des nouvelles perspectives sociales et bien souvent, une réussite économique.

Dans l'espace frontalier franco-brésilien, un autre groupe religieux important est celui originaire des croyances et des rites africains amenés par les Noirs en esclavage en Amérique. Ils ont pu maintenir, jusqu'à l'époque actuelle, leurs croyances, souvent en les dissimulant derrière un masque chrétien, devenu sous certains aspects, syncrétiques, dans le monde caraïbe et latinoaméricain. Les dieux des cultes d'origine africaine survivent sous la forme de Candomblé et Tambor de Mina au Brésil, de la Santeria à Cuba et du Vaudou ${ }^{6}$ en Haïti et en Louisiane. Par ailleurs, ils ne cessent d'émigrer d'une région à une autre, en se multipliant et intégrant des sectes. Ils continuent à s'adapter aux mutations du milieu et des sociétés.

En Guyane, comme d'ailleurs dans toute la Caraibe, Vaudou haïtien, Umbanda et Candomblé brésilien connaissent une vogue grandissante. Pour les pouvoirs publics, ce n'est pas toujours facile de répertorier ces mouvements comme étant simplement des expressions de foi et non pas comme de nouvelles sectes. Ces croyances sont encore combattues par les autorités religieuses monothéistes mais aujourd'hui, ces croyances sont surtout la cible des nouvelles églises chrétiennes. Vaudou, Umbanda ${ }^{7}$ et Candomblé, parmi d'autres croyances d'origine africaine et indienne, telle que la Pajelança ou Cura, étaient longtemps cantonnés dans les couches populaires. Désormais, ce sont aussi les classes bourgeoises, les intellectuels en quête de sens, des hommes d'affaires, des artistes et des femmes du monde que se sentent attirés par ces pratiques considérées souvent comme primitives ou sataniques. Il serait intéressant de poser la question dans une autre étude, sur les motivations qui poussent certains individus dits instruits et certaines classes sociales fortunées, vers ces rituels, considérés par beaucoup comme étranges. Il resterait aussi à savoir s'il agit, pour les nouveaux adeptes de ces rituels, d'une fascination morbide, un attrait de l'exotisme, un engouement pour le surnaturel ou une nostalgie du divin'.

\section{Conclusion}

L'espace amazonien défie tous les modèles, toutes les structures rigides et ne demande qu'à se situer dans une troisième voie possible. Cette voie est celle de la forêt, des fleuves et de ses habitants, dans le respect d'un espace protégé et respecté. De la diversité culturelle et cultuelle, l'Amazonie apparait, malheureusement comme une mosaïque, une juxtaposition de communautés. En Guyane, par exemple, compte aujourd'hui environ 9000 Amérindiens répartis en six ethnies: les Arawak, Kali'na, Palikur, Teko, Wayampi et Wayana. Chaque ethnie possède sa langue

\footnotetext{
${ }^{6}$ Le vaudou est l'adaptation par le Fon d'un mot yoruba signifiant « dieu ». Il est né de la rencontre des cultes traditionnels des dieux yorubas et des divinités fon et éwé, lors de la création puis l'expansion du royaume fon d'Abomey aux XVIIe et XVIIIe siècles. Le vaudou est le fondement culturel des peuples qui sont issus par migrations successives du Togo actuel, les Adja, dont les Fons, les Gouns, les Ewé, et dans une certaine mesure les Yorubas.

${ }^{7}$ L’Umbanda, d'origine afro, a des racines Jéjé, Nago, Malé, Bantou, Mina. Dans sa forme actuelle, c’est un mélange syncrétique avec le kardécisme, la pajelança et le catholicisme

${ }^{8}$ AM magazine, Afrique Magazine, section Histoire d'Iles, article, «Les possédés de la Caraibe », apparu le 01/10/99, Paris. Édition numérique : http://www.ecaraibes.com/article/article.asp?id_article $=11194345625$
} 
et sa culture. On peut aussi distinguer les Amérindiens de l'intérieur des terres et les Amérindiens du littoral. Les wayampi de Guyane vivent sur le moyen et le haut Oyapock et un groupe wayampi vit au Brésil dans la Terre indigène wayampi, dans l'état de l'Amapá. Les Teko quant à eux, sont repartis entre le bassin de la rivière Camopi, affluent de l'Oyapock (TRITSCH, 2013). Même si le métissage, l'immigration est de plus en plus

fréquente en Guyane, il existe peu d'espace qui permette un rapprochement entre les respectives communautés amazoniennes ou périphériques avec le noyau central, Paris et Brasília.

Les pouvoirs publics, les moyens de communication, les responsables des communautés religieuses ont peut-être conscience de la variété culturelle de l'Amazonie. Aussi, savent-ils peutêtre qu'il faut favoriser l'intégration des diverses ethnies amazoniennes. Pour les immigrés, l'intégration devient difficile dans la mesure où il semble insurmontable de dépasser les stéréotypes que le noyau central souvent véhicule à travers les médias. Aux stéréotypes s'ajoute le problème de communication puisque les immigrants, ont du mal à parler les langues dites officielles. Enfin, l'homme amazonien, en raison de ces vagues migratoires récentes, sont invités à définir ou à redéfinir leur être amazonien et leur rapport à l'autre, au monde et à leur passé. Ce processus d'intégration peut être positif à condition que le rapport centre-périphérie ne soit pas simplement un processus d'acculturation passive ou même une absorption par une culture dominante (BLÉRALD, 2007).

Cette absorption peut aussi être vécue comme une invasion, une agression de la part de la périphérie. Dans ce rapport centre-périphérie ce qui est en question c'est l'autre, l'altérité. Dans cette perspective l'ensemble d'images et représentations se trouvent faussées. Derrière le fantasme de l'invasion pour des raisons économiques ou la domination culturelle des grands centres métropolitains se cachent les problèmes d'ordre socio-économique et socioculturel. Pour l'instant, en Amazonie, le facteur religieux n'est pas soulevé, d'ailleurs, il n'a pas raison de l'être, car les croyances afro-amérindiennes puisent dans les mêmes sources. Enfin, dans cet espace amazonien, isolé par le milieu naturel, l'homme amazonien doit trouver un consensus et un état d'âme apaisé pour le vivre ensemble. À vrai dire, ce ne sont pas les garimpeiros ou les immigrants à la recherche d'une vie meilleure qui représentent une menace pour la société. La vraie menace se trouve dans le travers de la mondialisation/globalisation aux portes de l'Amazonie, juste mue par le profit et la richesse.

\section{Références}

ABRIC, J.C. Jeux, conflits et représentations sociales : thèse d'État. Aix-en-Provence - França: Université de Provence, 1976.

BLÉRALD, M. Identité et perspectives d'évolution des traditions créoles de Guyane:Comprendre la Guyane d'aujourd'hui. Matoury, Guiane Française: Ibis Rouge Éditions, 2007.

CALMONT, A. Immigration et développement en Guyane depuis la départementalisation: L'histoire de la Guyane depuis les civilisations amérindiennes Matoury, Guiane Française: Ibis Rouge éditions, 2006.

FATH S. Les protestants évangéliques français: La corde raide d'un militantisme sans frontière. Paris: Études, 2005.

GOMES, B. Les évangéliques au Brésil: stratégies territoriales et participation politique. Hérodote: Revue de géographie et géopolitique, Paris, v. CXIX, n. 4, 2005.

FOUCK, S. M. L.Comprendre la Guyane d'aujourd'bui. Matoury, Guiane Française: Ibis rouge éditions, 2007.

Histoire de la Guyane contemporaine, 1940-1982: les mutations économiques, sociales et politiques. Paris: Éditions l'Harmattan, 1992.

Histoire de la société guyanaise: les années cruciales:1848-1946. v. II. Paris: Éditions Caribéennes, 1987. 
Histoire générale de la Guyane française des débuts de la colonisation à l'aube de l'an 2000: les grands problèmes guyanais: permanence et évolution. Matoury, Guiane Française Ibis Rouge Éditions, 1996.

- La Guyane francaise au temps de l'esclavage, de l'or et de la francisation (18021946).Matoury, Guiane Française: Ibis Rouge Éditions, 1999.

MARCHAND-THEBAULT, M.R. L'esclavage en Guyane française sous l'ancien régime. Revue Francaise D'bistoire D'outre-mer, Paris, tome XDVII, n. 166 , 1960.

SCOT, J.P. Liberté-égalité-laïcité: Genèse, caractères et enjeux de la loi de 1905. Cabiers d'bistoire. Revue d'bistoire critique. Paris, n. 100, 2007.

TRITSCH, I. Dynamiques territoriales et revendications identitaires des Amérindiens wayäpi et teko de la commune de Camopi: Guyane française. 2013. 438 f. Tese (Doutorado) - Curso de Géographie, Université Des Antilles-guyane, Pointe-à-pitre, Guadaloupe, 2013.

Envio: 30/10/2019

Aceite: 08/11/2019 